CORRECTION

\title{
Author Correction: MicroRNA-302a promotes neointimal formation following carotid artery injury in mice by targeting PHLPP2 thus increasing Akt signaling
}

Ying-ying Liu', Xiu Liu' ${ }^{1}$, Jia-guo Zhou ${ }^{1}$ and Si-jia Liang ${ }^{1}$

Acta Pharmacologica Sinica (2022) 43:1879-1880; https://doi.org/10.1038/s41401-021-00815-0

Correction to: Acta Pharmacologica Sinica https://doi.org/10.1038/ s41401-020-0440-4, published online 21 July 2020

The authors apologized that the representative images of scratch assay and Transwell analysis (Fig. 5f, g) appeared incorrectly.
The correct images are presented. The authors declare that these corrections do not change the results or conclusions of this paper. The authors apologize for any inconvenience caused to the journal and readers. ${ }^{1}$ Department of Pharmacology, and Cardiac \& Cerebral Vascular Research Center, Zhongshan School of Medicine, Sun Yat-Sen University, Guangzhou 510080, China
Correspondence: Si-jia Liang (liangsj5@mailsysuedu.cn)

Published online: 1 December 2021 
a

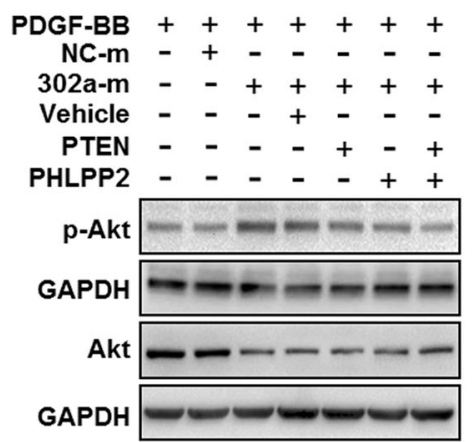

d

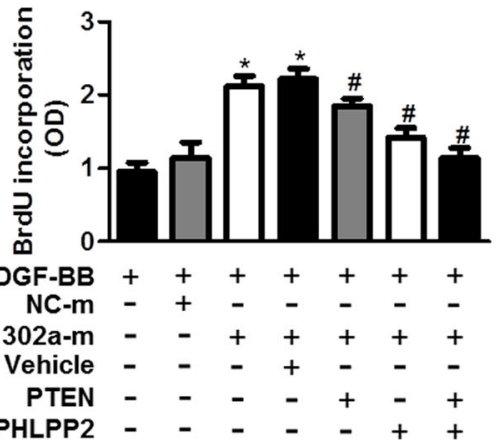

b

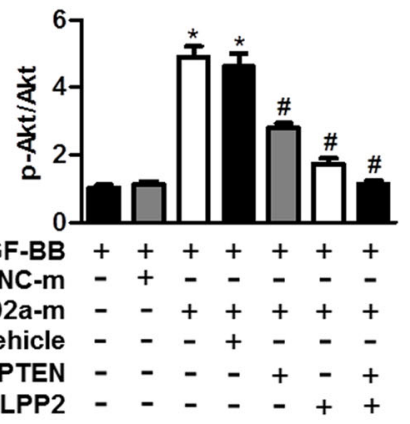

C

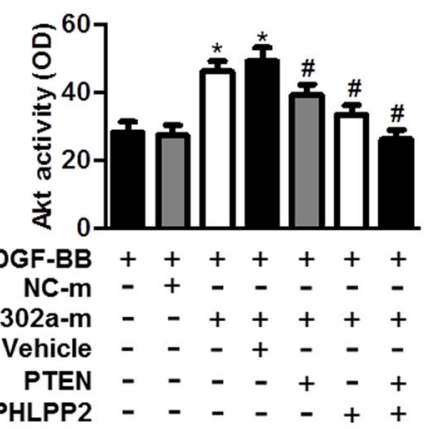

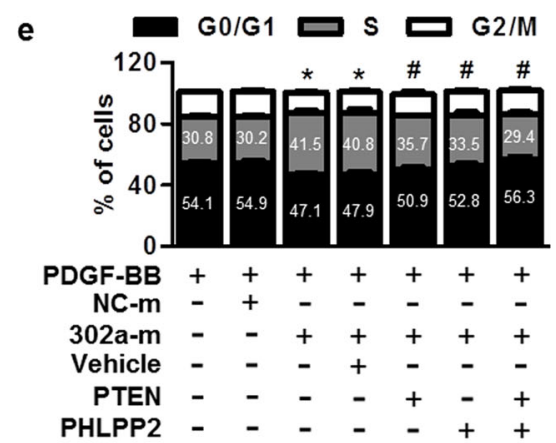
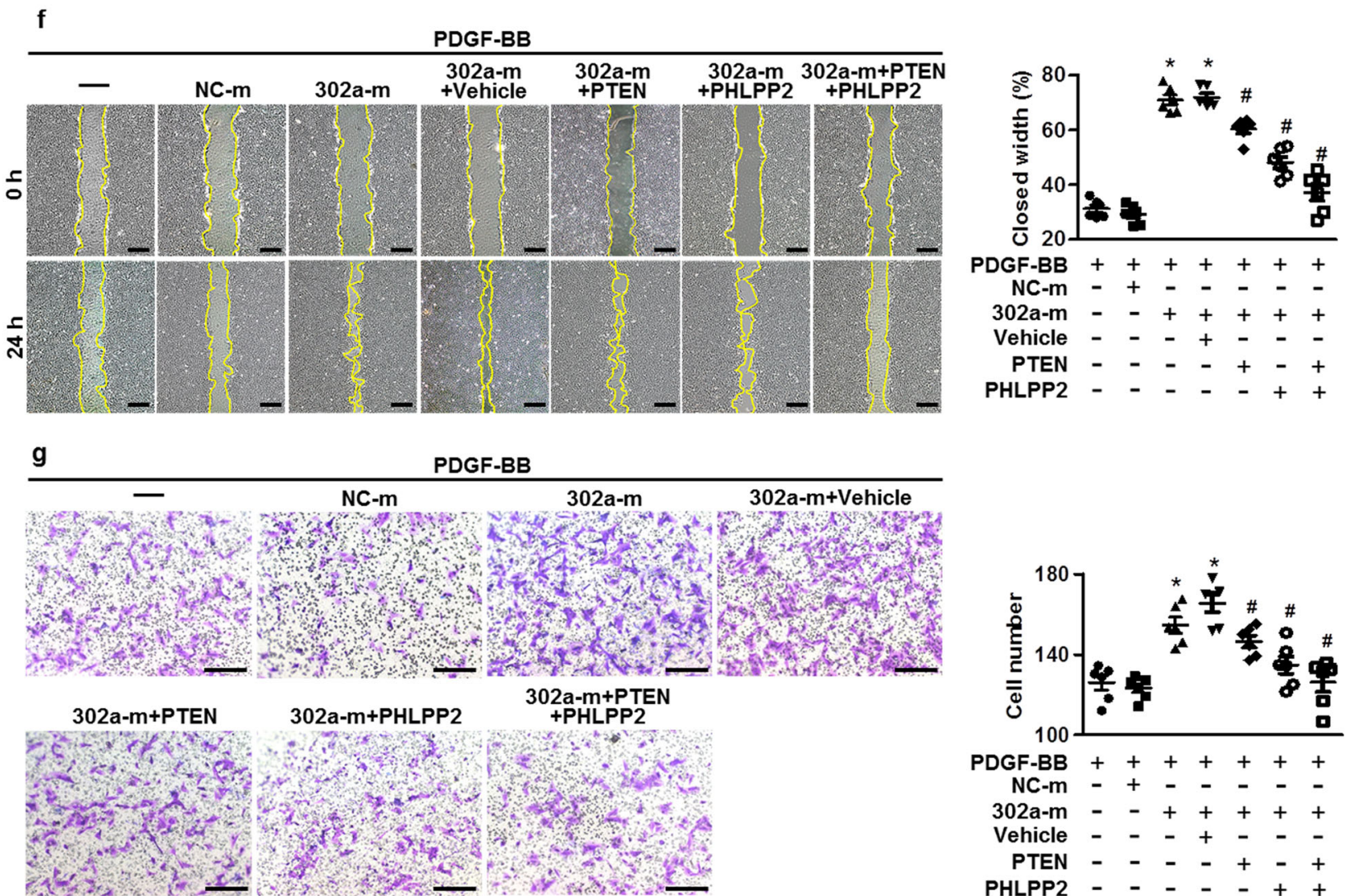

Fig. 5 Restoration of PHLPP2 or PTEN expression attenuates the effects of miR-302a on Akt activation, MASMC proliferation, and migration. a-c The cells were cotransfected with miR-302a mimics and pCMV vector (vehicle), PTEN plasmid, or PHLPP2 plasmid for $24 \mathrm{~h}$ and then treated with PDGF-BB for another $1 \mathrm{~h}$. Representative Western blotting images for $\mathrm{p}$-Akt and Akt are shown (a). The ratio of p-Akt to Akt (b) and Akt activity (c) were measured. Cell viability (d) and cell cycle (e) analyses after PDGF-BB treatment for $24 \mathrm{~h}$. Cell migratory capacity was examined by the scratch assay (f) and Transwell analysis (g). Scale bar, $200 \mu \mathrm{m}$ for the scratch assay and $100 \mu \mathrm{m}$ for the Transwell analysis. ${ }^{*} P<$ 0.05 vs. PDGF-BB, ${ }^{\#} P<0.05$ vs PDGF-BB $+302 \mathrm{a}-\mathrm{m}, n=6$. 